\title{
ENFERMERÍA PSIQUIÁTRICA COMUNITARIA EN SALUD MENTAL DE ALAVA «DEL HOSPITAL... A LA COMUNIDAD»
}

\author{
FABIOLA GASIAIN SAENZ \\ Centro Salud Mental Aranbizkarra \\ ANA I. RAmírez GorostizA \\ S.Alcoholismo y Ludopatías \\ Edurne Zurbano SAENZ \\ Centro Salud Mental. San Martín
}

\section{RESUMEN}

En el presente trabajo desarrollamos cronológicamente la evolución habida en el abordaje en la Salud Mental de Alava, así como la evolución paralela que ha supuesto para la profesión de Enfermera Psiquiátrica. Desde la creación del Asilo Provincial de Alava en 1925, donde tenían cabida entre otros, los enfermos mentales, hasta el momento actual, la implicación como profesionales que ejercemos nuestro trabajo en este ámbito, nos ha llevado a una reflexión sobre esta evolución que no solo ha impregnado lo institucional, sino que además ha posibilitado que surja la aparición de nuevos roles para la Enfermería en Salud Mental en la Comunidad

\section{COMMUNITY PSYCHIATRIC NURSING IN MENTAL HEALTH, IN ALAVA «FROM HOSPITAL TO...THE COMMUNITY»}

\section{SUMMARY}

The present study chronologically develops the evolution of mental health care in Alava together with the influence this mental health evolution has had in the Psychiatric Nursing profession.

Since the opening of the Alava's Asilo Provincial (Home), in 1925, where different kind of patients - including mental health patients - received attention, to the present time, those professionals who have worked withing the Home premises, have discovered how care evolution towards these sort of patients has influenced not only the institutional procedures but how it has rendered easy as well the appearing of new roles for the Mental Health Nursing in the Community.

\section{INTRODUCCIÓN}

Desde el cambio estético de nomenclatura de Asilo, auspiciado por organismos benéficos, a Hosjital Psiquiátrico, asumido por la Sanidad Pública y que contemplaba también la Atención Extrahospialaria Comunitaria, se ha producido un proceso diaámico que ha impregnado, como no, a los profesionales implicados en él. Se pasa de un entorno aospitalario donde la labor de la enfermera psiquiá- trica estaba mas o menos definida, a un nuevo emplazamiento en los Centros de Salud Mental donde, reflexivamente nos cuestionábamos un cambio en nuestro sistema de trabajo. No podíamos convertirlo en una reproducción de la actividad hospitalaria y tuvimos que reelaborar y reconstruir una nueva alternativa.

La relación enfermera-paciente no se da en un espacio vacío, sino en un lugar determinado y con un marco terapéutico diferente. La configuración de 
este, en nuestro caso fuera del hospital y ubicado en la comunidad, fue un punto de partida para reflexionar sobre nuestra practica y con ello "Construir un Nuevo Conocimiento Profesional".

Fuimos protagonistas de este Proceso de Desarrollo Profesional no sin olvidarnos de la carga de incertidumbre que conllevaba. Teníamos a nuestro favor el hecho de ser conscientes de una necesidad de cambio, una gran motivación personal y profesional y una serie de factores propicios que desde la propia institución favorecieron este proceso.

\section{HISTORIA DE LA SALUD MENTAL Y ENFERMERÍA PSIQUIATRICA DE ALAVA}

En el territorio histórico de Álava y en su capital, Vitoria, se comenzaron los trámites para la elaboración de un proyecto de construcción del "Asilo Provincial de Santa $M^{a}$ de Las Nieves" en el año 1899, haciéndose cargo Diputación de la construcción del edificio que constaría de dos partes bien diferenciadas, la correspondiente al "Asilo de Pobres" y la del pabellón de "Alienados". En el "Reglamento del Asilo de Santa $M^{a}$ de las Nieves" elaborado en 1.926 se describía el objeto de este asilo como: "Proporcionar albergue, vestido y alimentación a los pobres desvalidos y expósitos de ambos sexos, asistiéndoles en sus enfermedades mentales y comunes".

En el apartado referido al personal sanitario de la institución se hacía mención de los siguientes profesionales:

- Un médico director técnico en la asistencia de enfermos y dementes.

- Un practicante ayudante del médico.

- Los vigilantes que la atención del manicomio exigía.

- Personal dedicado a la labor de intendencia y limpieza.

- Una comunidad de Religiosas Hermanas de la Caridad, bajo la dirección de la superiora.

En lo relativo a funciones del practicante, señalaban las siguientes:

"Para el servicio de las secciones de hombres de asilo manicomio, y también para las secciones de mujeres, cuando el médico lo considere necesa- rio, en cuyo caso ejercerá sus funciones acompañado por una hermana de la caridad, habrá ur practicante que residirá en el establecimiento y de. penderá inmediatamente y estará a las órdenes de. médico-director".

Se consideraban obligaciones del practicante:

- Acompañar al médico en las visitas

- Practicar todas las operaciones de cirugíc que el médico prescriba, y ayudar a este en la: operaciones que efectúe

- Administrar por si o por medio de los vigilan. tes y Hermanas de la Caridad, a quienes di. rija, los medicamentos y servicios hidroterápi. cos que haya dispuesto el facultativo

- Aplicar las sangrías y las sanguijuelas, admi. nistrar los baños, duchas, tópicos, medio: electroterápicos y extraer muelas

- Dirigir y presenciar el baño general, y el re. gistro al que están sometidos los alienados a ingreso

- Prestar asistencia a toda hora de la noche qut sea requerido por los encargados de la vigi. lancia, avisando al médico si lo cree necesa. rio

- Prestar además los servicios propios de sl profesión que la Junta encomiende.

Es preciso recordar "nuestros previos", de donde partimos y que bagajes impregnaban nuestrc profesión. Hay que subrayar que la enfermería a] cuidado de los enfermos mentales ha estado en manos de órdenes religiosas. En 1979 en el Asilo había seis enfermeras religiosas sin especialidad. Este hecho ha estigmatizado de entrada nuestros roles profesionales, los puestos de responsabilidad recaían en la superiora de la comunidad que era a la sazón la jefa de enfermeras y en las supervisoras que también eran religiosas y hasta 1986 no se nombra como responsable de enfermería a una profesional con especialidad que no perteneciese al clero. En lc que a nuestra formación se refiere, se instauró en la "Residencia Provincial" (nombre que adquirió el Asilo con el paso de los años) la Especialidad de A. T. S. Psiquiátrica, la primera promoción surge en el curso 1977-78 y existe hasta la promoción del 86. 
siendo en 1981 cuando comienzan a trabajar las primeras Enfermeras Psiquiátricas no religiosas.

En 1991 se creó en la Escuela Universitaria de Enfermería de Álava el curso Postgrado en Salud Mental para llenar el vacío provocado por la falta de la Especialidad.

La apertura ha sido lenta y progresiva, Ilegando a ser en la actualidad 66 enfermeras trabajando en Salud Mental.

Los grandes cambios en el abordaje de la enfermedad mental en la provincia ocurrieron en la dézada de los 80. Por un lado se desarrolla la Atención Psiquiátrica Extrahospitalaria en Álava entre los años 83 y 85 y en el 84 se crea una unidad de osiquiatría en el hospital General Santiago Apóstol, oor otro con la Ley General de Sanidad del 25 de Abril de 1.986 la Salud Mental fue asumida por el Sistema Nacional de Salud y por último en Enero de 1988 se integran todos los recursos en el Servizio Vasco de Salud, Osakidetza.

En Febrero de 1985 se crearon las cuatro prineras plazas de Enfermeras Psiquiátricas Comuniarias. Fuimos las primeras profesionales a tiempo otal en un Centro de Salud Mental (C.S.M), el reso de los profesionales acudían uno o dos días por ;emana a las consultas extrahospitalarias. Estas zuatro plazas correspondían a los cuatro primeros Z.S.M. de Vitoria.

A partir de 1987 se fueron creando nuevos dissositivos en la Red de Salud Mental de Álava, que in la actualidad se compone de:

-5 Centros de Salud Mental

- Unidad de Psiquiatría Infantil

- Hospital de Día

- Servicio de Alcoholismo y Ludopatias

- Centro de Tratamiento de Toxicomanías

- Servicio de Rehabilitación Comunitaria

\section{JEL HOSPITAL... A LA COMUNIDAD}

La atención Psiquiátrica Extrahospitalaria en Ílava, surgió en los inicios de los años 80, se conenzó colaborando de forma voluntaria con la paricipación multidisciplinar de profesionales que rabajábamos en el interior del Hospital Psiquiátrio y que a tiempo parcial y en mini-equipos, sali- mos "extramuros" para ubicarnos en "los barrios", utilizando para ello despachos de parroquias, locales de la asociación de vecinos etc., donde ofertábamos un día por semana nuestro hacer profesional, compatibilizando esta actividad con nuestra jornada laboral en el Hospital Psiquiátrico.

Lo que comenzó siendo una experiencia a pequeña escala, se convirtió en un corto espacio de tiempo, en las bases de la actual Atención Psiquiátrica Extrahospitalaria de Álava.

Los profesionales de Enfermería Psiquiátrica en el momento en que surgía este modelo de sectorización, año 1982, convencidos del futuro de esta "innovadora" idea, colaboramos desde el principio en el desarrollo de estas actividades.

Los inicios en la comunidad no fueron fáciles, soportamos tensiones, dificultades y obligaciones que ahora con el tiempo nos producen cierta sonrisa (buscar piso para ubicar las consultas, hacer de secretarias, telefonistas, recadistas...)

Hemos pasado por diversas fases; estábamos formadas y entrenadas para enfrentarnos cotidianamente al trabajo hospitalario y tuvimos que esforzarnos en hacernos reconocer profesionalmente, pero ahora nuestro continente se ha transformado, ya no nos movemos entre habitaciones, botiquines y comedores, ya no preparamos medicación ni extraemos sangre. Ahora nos enfrentamos a nuestro trabajo en "un despacho", sin uniforme y con "el solo" instrumento de la escucha y la palabra.

Efectivamente nuestro rol tenía que cambiar y para ello hemos necesitado de unos pilares básicos continuos:

1.- El mantenimiento periódico de reuniones profesionales de Enfermeras Psiquiátricas Extrahospitalarias que hemos realizado desde el comienzo de nuestro trabajo en el Extrahospitalario, en un principio se realizaban con una frecuencia semanal y en la actualidad mensual.

Estas reuniones surgieron como una "necesidad" donde se vehiculizaban tensiones, angustias y proyectos. En los inicios de nuestro trabajo extrahospitalario tenemos que reconocer que estas reuniones cumplieron cierta función "terapéutica". En la actualidad, solemos hacer unas 10 reuniones al año y con una duración de hora y media. El conte- 
nido de estas reuniones aborda temas profesionales donde se comentan las informaciones que aportan las representantes de enfermería en las diferentes Comisiones (Calidad, Protocolos, Docencia, Bioética, etc.) y se trasmiten las novedades que con relación a Salud Mental van surgiendo.

Otra de las funciones de estas reuniones ha sido el ser un marco adecuado para la integración y reciclaje de las nuevas profesionales de enfermería que se han ido incorporando a la Red de Salud Mental Extrahospitalaria de Álava.

\section{2.- El interés por la "Formación Continuada y la Docencia".}

Un grupo de Enfermeras Psiquiátricas, empezamos impartiendo docencia teórica y práctica a las enfermeras que estaban cursando la especialidad de Psiquiatría Posteriormente hemos colaborado con la Escuela Universitaria de Enfermería de Osakidetza, realizando iguales funciones en los cursos Postgrado en Salud Mental y de Enfermería General organizados por dicha escuela, así mismo supervisamos las tutorías a las estudiantes de Enfermería Básica, que realizan prácticas en nuestros centros.

Hemos participado también, dentro de las Campañas anuales de Educación para la Salud del Ayuntamiento de Vitoria, impartiendo charlas en Centros Cívicos, en zonas rurales, etc.

La mayoría de la Formación Continuada la hemos realizado junto a las enfermeras del Hospital Psiquiátrico y estos últimos años ha ido dirigida sobre todo a "Metodología de Cuidados de Enfermería en Salud Mental", Además de cursos dedicados al reciclaje en Psicopatología, Psicofarmacología, Bioética, Calidad, etc.

Otra parte del colectivo ha realizado una Formación Específica en temas como Psicoanálisis, Bioética, Terapia de Familia, Psicoterapia de Grupo...

\section{NUEVOS ROLES EN ENFERMERÍA EN SALUD MENTAL EXTRAHOSPITALARIA}

Hoy, con más de trece años de trayectoria como Profesionales de la Enfermería Psiquiátrica Extrahospitalaria, hacemos un balance de lo que en su momento fueron posibilidades y hoy se han conver- tido en realidad. Para empezar a hablar de las funciones que desarrolla la Enfermera Psiquiátrica Co. munitaria, la palabra que mejor puede definirlas es la de proporcionar "cuidados de enfermería" ba sados en una metodología y desarrollados a través de un proceso terapéutico.

Ubicados en un contexto comunitario y dentrc de la faceta asistencial de nuestro trabajo, destaca. ríamos como una de las nuevas aportaciones que venimos desarrollando, la de "recibir" al paciente La Enfermera Psiquiátrica Comunitaria, posee la formación, experiencia y capacidad necesarias par: llevar a cabo la labor de recepción, valoración y re cogida de datos en lo que sería la primera entrevis. ta del paciente en el Centro de Salud Mental y ela borar y presentar el caso en la reunión de equipo donde se debate que profesional y que proyecto te rapéutico sería el más conveniente para él. Esta pri mera entrevista tiene como objetivo establecer e primer contacto, que es relevante en su trayectori: terapéutica, además se conoce y prioriza la deman da, se valora el estado general del paciente, tanto es su aspecto psíquico y físico como social, se reco gen datos significativos de la vida familiar, social : laboral. Por último se le explica el funcionamient del centro y la composición del equipo que va : atenderle.

Este proceso de "acercamiento" anuncia el co mienzo de una relación profesional. La enfermer: debe dotar a esta primera entrevista de un clima di privacidad, confidencialidad y profesionalidad. $\mathrm{L}$ : consecución de todos estos objetivos constituye 1 : realización de "La Consulta de Acogida"

Además de esta función de acogida, otra activi dad innovadora que desarrollamos es la que tien que ver con la "Relación con otros dispositivo asistenciales de la Red de Salud Mental", mediant "Las Visitas de Coordinación" con las "Áreas dı Hospitalización" del Hospital Psiquiátrico y de li planta de psiquiatría del Hospital General, así com con las "Estructuras Intermedias" donde se inclu yen visitas al Hospital de Día, Taller Ocupacional : servicio de Rehabilitación Comunitaria. El objetivi es el de buscar "La Continuidad de Cuidados" es los diversos dispositivos en los que puede ser aten dido el paciente. 
Las reuniones se realizan de una forma periódica (semanal o quincenalmente) mediante el desplazamiento de una de las enfermeras del Centro de Salud Mental a las estructuras enunciadas anteriormente, y mediante reuniones ya programadas, intercambiar con las enfermeras psiquiátricas de planta y del resto de dispositivos, la información sobre evolución y pautas a seguir en relación con los pacientes ingresados.

Otra de las actividades que conlleva el trabajo en la comunidad es la disponibilidad por parte de la enfermera psiquiátrica para ser en un porcentaje muy elevado de ocasiones, la persona dentro del equipo de Salud mental elegida para realizar "Las Visitas a Domicilio", estas tienen un carácter peculiar o diferente a las que se realizan desde los centros de Atención Primaria. Nos sirven para valorar "in situ " la situación, siempre deben ser pactadas de antemano, el paciente debe conocer nuestra visita para evitar en lo posible crear situaciones de suspicacia, temor o sensación de invasión de su intimidad, sobre todo en las visitas domiciliarias dirigidas a pacientes con patologías psicóticas y riesgos suicidas. Las programadas para el cuidado de personas con dificultades motoras para desplazarse al centro (ancianos, encamados etc.), deberán cumplir las anteriores premisas, pero no conllevan especiales dificultades.

Lo que también consideramos fundamental y que impregna todas las actividades de Enfermería en Salud Mental es "La Educación para la Salud", en la cual intentamos responsabilizar a los pacientes, familia y comunidad del mantenimiento y cuidado de su salud mental, prestando atención a sus problemas, comportamientos, creencias y actitudes.

La educación sanitaria dirigida también a la comunidad se realiza de manera tanto formal como informal y va dirigida a aportar información, buscando un cambio de actitudes y/o comportamientos. Por tanto la educación sanitaria va dirigida tanto al individuo como a la comunidad y en ocasiones se realiza un apoyo y asesoramiento a instituciones, tales como las "Asociaciones de Familiares de Enfermos Psiquiátricos", "Enfermos de Alzheimer, demencia senil etc. y al Ayuntamiento en las campañas que realiza de Educación Sanitaria.
Además de estas actividades específicas de la comunidad, realizamos otras compatibles con el marco hospitalario, como son el control, según protocolo, de tratamientos farmacológicos ( Clínica de Litio, Leponex, Carbamacepina) etc., Psicoterapias-Cooterapias (individuales, familiares y grupales), estas funciones solo las realizan enfermeras con formaciones especificas en las diversas técnicas psicoterapeúticas y que están entrenadas y preparadas para ello.

A la función asistencial tendríamos que añadir las funciones Docentes, Investigadoras y Administrativas tan ligadas a nuestra profesión pero que no son objeto de desarrollo en este trabajo.

\section{BIBLIOGRAFÍA Y FUENTES DOCUMENTALES}

Lejarreta, J. (1.930). Reglamento del Asilo Santa María de Las Nieves, Alava.

Bases técnicas para la Reforma de la Atención Psiquiátrica y la prevención la Salud Mental en la Comunidad Autónoma del País vasco.

(DASGV) (1990) Servicio de Salud Mental: Plan Estratégico. Departamento de Sanidad y Consumo. Dirección de Asistencia Sanitaria. Gobierno Vasco.

Rosette Poletti R.(1.990). Cuidados de Enfermería. Tendencias y conceptos actuales. Ediciones Rol, Barcelona 